\title{
The mRAD18Sc/HR6B protein complex localizes to replication foci and UV induced DNA lesions
}

\section{$\underline{\text { Roald van der Laan }}{ }^{1}$, Henk P. Roest ${ }^{1}$, Willy M. Baarends ${ }^{2}$, Evert-Jan Uringa ${ }^{2}$, Gert A. van Cappellen $^{2}$, J. Anton Grootegoed ${ }^{2}$ and Jan H.J. Hoeijmakers ${ }^{1}$}

${ }^{1}$ MGC-department of Cell Biology and Genetics, Center for Biomedical Genetics; and ${ }^{2}$ Department of Endocrinology and Reproduction, Erasmus Medical Center Rotterdam, P.O. Box 1738, 3000 DR Rotterdam, The Netherlands

At least five multienzyme DNA repair pathways are currently known; one of these is termed postreplication repair (PRR). PRR facilitates tolerance of DNA damage during the duplication of the genome in S-phase cells overcoming termination of this process when DNA damage is present.

S. cerevisiae RAD18 encodes a DNA binding protein that contains, in addition to a $\mathrm{ZN}$-finger domain, also a RING-finger domain. The latter domain is a hallmark of a family of ubiquitinligating enzymes (E3) involved in the ubiquitin-conjugation pathway. This possible function was confirmed by the fact that it interacts with the ubiquitin-conjugating enzyme (E2) RAD6. Both proteins were identified in a screen for radiation-sensitive mutants and function in the postreplication repair of DNA damage. Inactivation of RAD6 results, in addition to several features, in a severe UV sensitive phenotype. RAD 18 mutants show a similar sensitivity to UV irradiation, thereby linking these two proteins also in a biological way.

Mammalian homologs of RAD6, HR6A and HR6B and the murine homolog of RAD18, mRAD18Sc [1] interact, suggesting a similar function in mammals [2]. Here we show that HeLa cells stably expressing HR6B and mRAD18Sc fused to fluorescent markers co-localize in the nucleus. UV irradiation results in nuclear relocalization of YFP-mRAD18sc into prominent foci that fully colocalize with HR6B-CFP foci. At least a large fraction of these foci, represent sites of DNA damage. Both YFP-mRAD18Sc and HR6B-CFP co-localize with sites containing DNA damage in HeLa cells treated with local damage induced by UV irradiation. In undamaged cells both mRAD18Sc and HR6B reside in part in replication foci in S-phase cells and immunofluorescence experiments using antibodies against PCNA show striking co-localization with PCNA. Finally, we tested the interaction between YFPmRAD18Sc and HR6BCFP in living cells by photo-acceptor bleaching FRET (fluorescence resonant energy transfer) in locally irradiated areas of the nucleus of HeLa cells. We were able to visualize an increase in the relative CFP fluorescence intensity after bleaching YFP as a result of a disruption of the energy transfer from CFP to YFP (Figure 1.). This finding indicates that a significant fraction of YFPmRAD18Sc and HR6B physically interact in vivo.

To further investigate the properties of the mRAD18Sc-HR6B complex in living cells, we are currently studying the dynamics of both proteins utilizing sophisticated photobleaching protocols including FRAP/FLIP and also FCS. Finally, we will try to follow the complex formation in living cells by cross-correlation FCS and FLIM analysis in living cells.

References

1: R. van der Laan et al. Genomics 69. (2000); 86-94

2: S. Tateishi et al., Mol Cell Biol. 23(2). (2003); 474-81. 


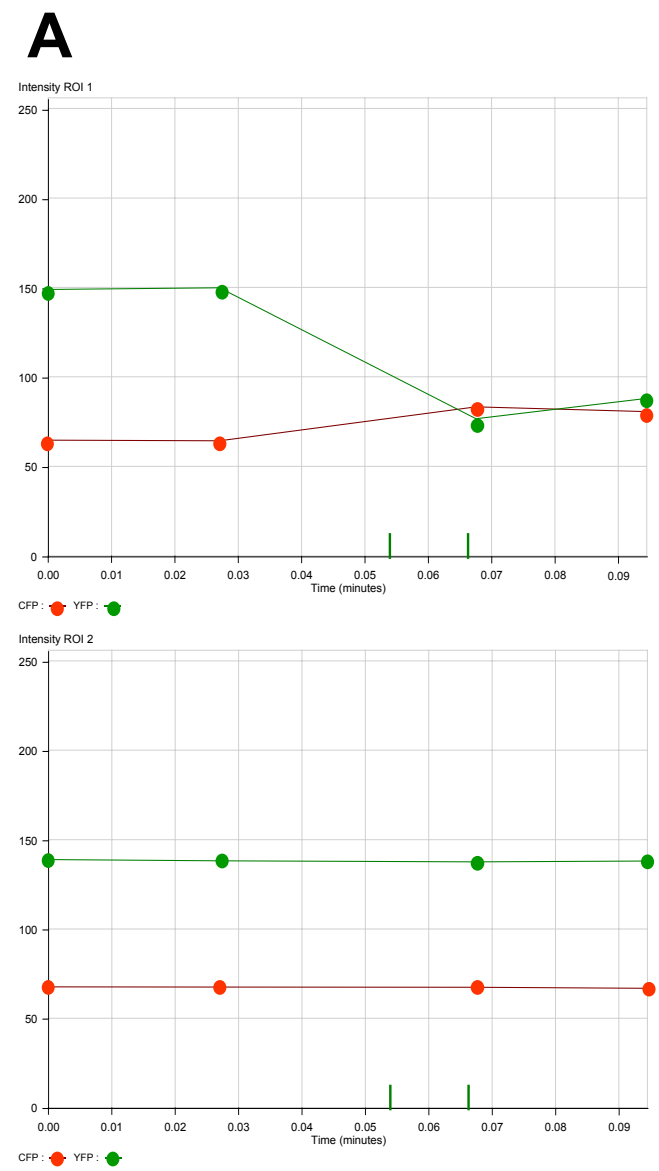

B
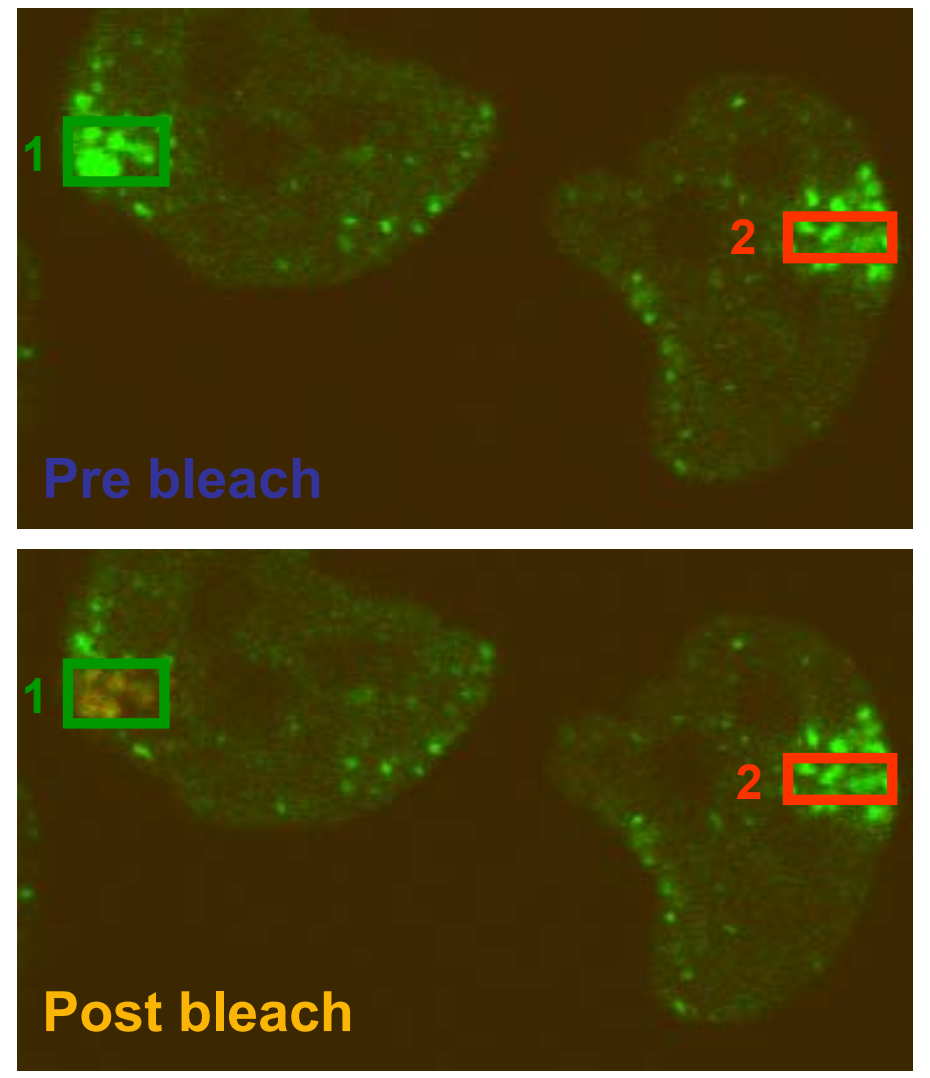

\begin{tabular}{|l|l|l|l|l|}
\hline Time $(\mathbf{m})$ & RO1CFP & ROI2YFP & RO1CFP & ROI2YFP \\
\hline 0.000 & 58.5 & 149.8 & 62.1 & 139.6 \\
\hline 0.027 & 58.1 & 150.9 & 60.5 & 139.0 \\
\hline 0.067 & 87.7 & 77.6 & 62.4 & 138.3 \\
\hline 0.095 & 81.6 & 88.9 & 59.6 & 138.8 \\
\hline
\end{tabular}

Figure 1.

Acceptor photobleaching FRET analysis of HeLa cells expressing YFPmRAD18Sc and HR6BCFP that were locally irradiated with $10 \mathrm{~J} / \mathrm{m} 2$ of UVC. A: pre- and post bleach image, the green rectangle is the bleached area and the red rectangle the control region; B: represents the relative YFP and CFP intensity for both regions in time; and $\mathrm{C}$ : a table showing the data presented in the graph in numbers with the pre bleached intensities in blue and the post bleached numbers in yellow. 